\title{
Development of an equation of state for the square-well chain molecules of variable well width based on a modified coordination number model
}

\author{
Jung-Chin Tsai ${ }^{1}$, Yan-Ping Chen* \\ Department of Chemical Engineering, National Taiwan University, Taipei 106, Taiwan
}

Received 12 September 2000; accepted 20 April 2001

\begin{abstract}
An equation of state (EOS) is developed according to the statistical associating fluid theory (SAFT) model. The dispersion term in the new EOS is derived by a modified coordination number equation. This modified coordination number expression gives correct results at low density and close-packed regions. It also agrees well with the computer simulation results for square-well (SW) fluids. A modified expression of the chain term compressibility factor for the SW fluid with variable well width is proposed in this study. Comparison of the calculated compressibility factors with molecular simulation data for tangent hard sphere chains and SW chains indicates that the new EOS yields satisfactory results and relatively smaller deviations than those from other equations. Finally, the new EOS is employed on real fluid systems of $n$-alkanes. For each pure fluid, four EOS parameters are regressed. These pure fluid parameters yield reasonable correlation with the molecular weight for $n$-alkanes up to $n$-eicosane. The pure fluid properties are well calculated by the new EOS. Prediction of the saturated vapor pressures of higher carbon number alkanes also shows satisfactory results. The new EOS developed in this study is consistent with the molecular simulation data for SW fluids with variable well width. It is simple in mathematical form, and is applicable from small molecular weight compounds to long chain molecules. (C) 2001 Elsevier Science B.V. All rights reserved.
\end{abstract}

Keywords: Equation of state (EOS); Theory; VLE

\section{Introduction}

The development of accurate equations of state from statistical mechanics, which can be used from small molecules to long chain fluids, receives much attention in recent studies. The statistical associating fluid theory (SAFT) equation of state (EOS) is an example, which was established based on the thermodynamic perturbation theory of polymerization [1-4]. The SAFT EOS has four terms: the hard

\footnotetext{
* Corresponding author. Fax: +886-2-2362-3040.

E-mail address: ypchen@ ccms.ntu.edu.tw (Y.-P. Chen).

${ }^{1}$ Present address: Department of Chemical Engineering, Minchi Institute of Technology, Taishan 243, Taipei, Taiwan.
} 
sphere repulsion force between segments; the attractive dispersion force; the chain formation contribution between segments and the polar attraction force between associating sites. Various versions of the SAFT type equations have been proposed in literature. Those equations used the modified forms of the chain formation and the dispersion terms for either the hard sphere or square-well (SW) chain molecules.

For example, Huang and Radosz [5] applied the SAFT EOS to real fluids of hard sphere chains. They used the polynomial equation of Alder et al. [6] for the dispersion term and the hard sphere pair correlation equation for the chain term. Fu and Sandler [7] modified the dispersion term of the SAFT EOS by using the coordination number equation of Lee et al. [8] to obtain the simplified SAFT EOS. Tavares et al. [9] employed the second-order Barker and Henderson perturbation theory for the dispersion term, and the first-order Wertheim thermodynamic perturbation theory for the chain formation term to derive an analytical EOS of SW chain fluids of variable well width. Gil-Villegas et al. [10] used the mean-value theorem to solve for the hard sphere radial distribution function in the dispersion term, and analyzed the chain molecules of four types of monomer potential functions with variable attractive ranges. An EOS for heterosegmented polymers was proposed by Adidharma and Radosz [11] where the second-order perturbation theory of Barker and Henderson with a truncation correction was used for the dispersion term. Various approximations of the SAFT type EOS for SW chains were compared by Adidharma and Radosz [12]. Generally, different combinations of the expressions for the dispersion and chain formation terms give satisfactory correlation of the saturated properties of real $n$-alkanes. It was also found that the inclusion of the dimer structure in the SW chain terms gave no significant contributions.

Another type of EOS for chain-like fluids is the perturbed-hard-sphere-chain (PHSC) EOS. Song et al. [13] used the modified Chiew's equation [14] for hard sphere chains as the reference term, and employed a simple van der Waals attractive form for the perturbation term. Hino and Prausnitz [15] simplified the analytical solution of Chang and Sandler [16] and obtained a modified theoretical perturbation term for the SW potential with variable width. Feng and Wang [17] replaced the perturbation term in the PHSC EOS by using a SW coordination number model, and correlated the vapor-liquid equilibria of polymer solutions and liquid-liquid equilibria for polymer blends. Recently, Kim and Bae [18] modified the chain term in the PHSC EOS using the form proposed by Chapman et al. [19]. A review for the development of cubic and non-cubic type EOS was given by Wei and Sadus [20].

The goal of this study is to develop a SAFT-based EOS which can be used for both the hard sphere and SW chain molecules of variable well width. A modified expression for the chain term compressibility factor of SW fluids is obtained by correlating the molecular simulation data. The dispersion term is expressed using a modified coordination number model with parameters that are also correlated from the molecular simulation data of SW fluids. This new EOS is simple in mathematical forms, and preserves the theoretical basis of SW chain fluids. This EOS is applied to real $n$-alkane fluids. Optimally fitted parameters are presented and the saturated properties are satisfactorily correlated. Applications of the generalized EOS parameters to predict the properties of other pure fluids are also presented in this study.

\section{Model development}

In the SAFT or PHSC type EOS, the compressibility factor of a non-associating chain fluid is written as the summation of a segment term and a chain term

$$
z=z^{\text {seg }}+z^{\text {chain }}
$$


The segment term consists of the compressibility factor of a hard sphere fluid $z^{\text {hs }}$, and an attraction term, $z^{\text {disp }}$. For a chain of $m$ segments, the compressibility factor is expressed as

$$
z=1+m z^{\text {hs }}+m z^{\text {disp }}+(1-m) z^{\text {chain }}
$$

The hard sphere term is written by the equation of Carnahan and Starling [21]

$$
z^{\mathrm{hs}}=\frac{4 \eta-2 \eta^{2}}{(1-\eta)^{3}}
$$

where $\eta$ is the reduced density defined as

$$
\eta=\frac{1}{6} \pi \rho \sigma^{3}
$$

The dispersion term in the SAFT type EOS represents the nonpolar attractive interaction between molecules. Typical expressions for the dispersion term are: (1) a polynomial argon fit [22]; (2) a secondorder Barker-Henderson perturbation form [9-11]. The perturbation terms are obtained from either the empirical correlation of molecular simulation data, or the analytical approximation by the Percus-Yevick method [16] and mean-value theorem [10]. The solution of Chang and Sandler [16] is completely analytic. The perturbation terms are usually complex functions that are tested with simulation data at a fixed width parameter for a SW fluid; (3) a much simpler van der Waals type equation $[13,18]$ or (4) an equation derived from a coordination number model [17]. In our previous study [23], we have derived a coordination number model based on the SW potential. That model is further modified in this investigation, and used to derive the dispersion term of the new EOS. For the SAFT type EOS, the chain term is written as a function of the radial distribution function as presented by Tavares et al. [24]. According to the suggestion of Anderson and Chandler [25] and Barker and Henderson [26], the radial distribution function for a SW fluid is expressed in a logarithm expansion. The leading term is the hard sphere distribution function, and the first-order perturbation term is a function of reduced density and temperature. The chain term for the compressibility factor is derived by the differentiation of the SW radial distribution function with respect to the reduced density. It is also a combination of the hard sphere contribution of the compressibility factor plus a correction term that is a function of reduced density and temperature. The correction part for the chain term of the compressibility factor is obtained in this study by correlating the molecular simulation data of the compressibility factors for hard sphere and SW chain fluids of variable well width and temperatures. A complete EOS is then formulated with capability of representing the hard sphere to SW chain fluids. The mathematical form of the resulting EOS is simple and easy for engineering applications.

\section{The modified coordination number model and the dispersion term for the SW fluids}

In this study, we develop the dispersion term from a modified SW fluid coordination number model based on our previous work [23]. For a SW fluid, the potential function is expressed as

$$
\Gamma(r)= \begin{cases}\infty, & r \leq \sigma \\ -\varepsilon, & \sigma<r<\lambda \sigma \\ 0, & r \geq \lambda \sigma\end{cases}
$$


where $\varepsilon$ is the energy depth, $\sigma$ a molecular size parameter and $\lambda$ a characteristic well width parameter. Yu and Chen [23] suggested that the coordination number model based on a SW potential should be written as

$$
N_{\mathrm{c}, \rho}=N_{\mathrm{c}, \rho=0} \Psi
$$

where $N_{\mathrm{c}, \rho}$ is the coordination number which is a function of the potential energy parameter and density, $N_{\mathrm{c}, \rho=0}$ the coordination number at a low density limit

$$
N_{\mathrm{c}, \rho=0}=\frac{4 \pi}{3} \rho \sigma^{3}\left(\lambda^{3}-1\right) \exp \left(\frac{\varepsilon}{k T}\right)
$$

$\Psi$ is a correction term which is expressed as a function of the reduced density and the energy parameter of the potential function. Various coordination number models have been presented in literature. As discussed in our previous study [23], several models are too complex, which result in difficulty of integration to obtain an EOS for fluid mixtures. Some boundary conditions must be satisfied for a theoretically correct coordination number model. First, Eq. (7) is approached at a low density limit. Second, the coordination number becomes independent of the energy parameter at a high density limit. For the SW fluid at the close-packed condition with $\lambda=1.5$, the coordination number is 18 . Third, for an equal-sized binary SW fluid mixture, the ratios of $N_{12} x_{2} / N_{22} x_{1}$ and $N_{21} x_{1} / N_{11} x_{2}$ approach to unity at the close-packed condition according to the molecular simulation results of Lee et al. [27]. Yu and Chen [23] developed an improved coordination number model, which agrees well with the computer simulation data. A modified perturbed hard sphere (MPHS) EOS was proposed by Yu and Chen [28], which gave satisfactory VLE calculation results for binary mixtures. The only drawback of the coordination number model developed by Yu and Chen [23] is that the close-packed limit was not satisfied. This deficiency can be improved by using a modified expression of the $\Psi$ correction term. The corresponding segment term of the EOS derived from the new coordination number model must also yield small deviation from the molecular simulation data for monomer SW fluids. In this study, a new coordination number model is written as

$$
\begin{aligned}
N_{\mathrm{c}}= & \frac{4 \pi}{3}\left(\lambda^{3}-1\right) \rho \sigma^{3} \exp \left(\frac{\varepsilon}{k T}\right) \\
& \times\left\{1+\left[1.28 \exp \left(-\frac{\varepsilon}{k T}\right)+A\left(\sqrt{2}-\rho \sigma^{3}\right) \exp \left(\frac{\varepsilon}{k T}\right)-1\right](1-\exp (1-\varphi))\right\}
\end{aligned}
$$

where

$$
\begin{aligned}
A & =0.25112-0.58939\left(\frac{\varepsilon}{k T}\right)+0.33225\left(\frac{\varepsilon}{k T}\right)^{2} \\
\varphi & =\frac{\sqrt{2}+\rho \sigma^{3}}{\sqrt{2}-\rho \sigma^{3}}
\end{aligned}
$$

According to the generalized van der Waals theory [29], the canonical partition function $Q$ is written as

$$
Q(N, V, T)=\prod_{i} \frac{1}{N_{i} !}\left(\frac{q_{\mathrm{r}, \mathrm{v}, \mathrm{e}}}{\Lambda^{3}}\right)^{N_{i}} V_{\mathrm{f}}^{N} \exp \left(-\frac{N \Phi}{2 k T}\right)
$$

where the mean potential $\Phi$ is determined using a coordination number model

$$
\Phi=k T \varepsilon \int_{T=\infty}^{T} \frac{N_{\mathrm{c}}(\rho, T)}{k T^{2}} \mathrm{~d} T
$$


The EOS is derived from the partition function by

$$
P=k T\left(\frac{\partial \ln Q}{\partial V}\right)_{N, T}
$$

Applying the new coordination number model, the dispersion compressibility factor is expressed as

$$
\begin{aligned}
z^{\text {disp }}= & \frac{2 \pi}{3}\left(\lambda^{3}-1\right) \rho \sigma^{3}\left\{\left[1+\frac{1.28 \varepsilon}{k T}-\exp \left(\frac{\varepsilon}{k T}\right)\right][\exp (1-\varphi)](1-\alpha)-\frac{1.28 \varepsilon}{k T}\right. \\
& \left.+\Omega\left((\exp (1-\varphi)-1)\left(\sqrt{2}-2 \rho \sigma^{3}\right)+\left(\sqrt{2}-\rho \sigma^{3}\right)[\exp (1-\varphi)] \alpha\right)\right\}
\end{aligned}
$$

where

$$
\begin{aligned}
& \alpha=\frac{\sqrt{2}(1-\varphi)}{\sqrt{2}-\rho \sigma^{3}} \\
& \Omega=\left[0.33225\left(\frac{\varepsilon}{k T}\right)^{2}-0.58939\left(\frac{\varepsilon}{k T}\right)+0.25112\right] \exp \left(\frac{2 \varepsilon}{k T}\right)
\end{aligned}
$$

Table 1 lists a comparison of various coordination number models. Most of them satisfy the low density limit condition. The additional requirements are not all met by other equations except Eq. (8) of this study. Table 2 compares the calculated coordination numbers at various reduced densities and temperatures for pure SW fluids at $\lambda=1.5$. It is shown in Table 2 that the GWL-II model gives the best results. The GWL-II equation is, however, too complex to derive an equation for fluid mixtures. The modified coordination number model shown in Eq. (8) yields comparably good results as those in our previous work and satisfies the close-packed requirement as well. Fig. 1 shows a graphical comparison of various coordination number models. The calculated results from Eq. (8) agree well with the molecular simulation data, and it is theoretically correct to derive the corresponding dispersion term for the EOS. Table 3 shows the calculated results of the compressibility factors for pure SW fluids at $\lambda=1.5$. Eight equations, with different expressions of the dispersion term, are compared in Table 3. It is shown that Eq. (14) gives good results with an overall absolute average deviation (AAD) of $<0.3 \%$ from the molecular simulation data. Fig. 2 presents a typical graphical comparison of our calculated results with the molecular simulation data for a pure monomer SW fluid. Fig. 3 shows a comparison of our calculated results for monomer SW fluids at various well widths and reduced temperatures. The agreement with the molecular simulation data is also satisfactory.

\section{Modification of the chain term compressibility factor}

The chain term of the EOS for SW fluids is related to the radial distribution function of the reference fluid at contact $g^{\text {ref }}(\sigma)$ by [24]

$$
\frac{a^{\text {chain }}}{R T}=(1-m)\left[\ln g^{\mathrm{ref}}(\sigma)-\ln g_{0}^{\mathrm{ref}}(\sigma)\right]
$$

where $a^{\text {chain }}$ is the chain term of the residual Helmholtz free energy, and $g_{0}^{\text {ref }}(\sigma)$ the radial distribution function at contact and at a limiting zero density. The SW fluid is taken as the reference fluid in this study. 
Table 1

Comparison of the coordination number models for pure SW fluids at the limiting states

\begin{tabular}{lllll}
\hline Model & Low density limit condition & $\begin{array}{l}\text { Close-packed } \\
\text { condition }(\lambda=1.5)\end{array}$ & $\begin{array}{l}\text { Mixture partition } \\
\text { function }\end{array}$ & $\lim _{\rho \sigma_{i j}^{3} \rightarrow \sqrt{2}}\left(N_{12} x_{2} / N_{22} x_{1}\right)=1$ \\
\hline Exact & $(4 \pi / 3)\left(\lambda^{3}-1\right) \rho \sigma^{3} \exp (\varepsilon / k T)$ & 18 & - & - \\
Lee-Sandler (LS) [8] & $12.73\left(\lambda^{3}-1\right) \rho \sigma^{3} \exp (\varepsilon / k T)$ & 18 & Yes & No \\
Lee-Chao (LC) [30] & $(4 \pi / 3)\left(\lambda^{3}-1\right) \rho \sigma^{3} \exp (\varepsilon / k T)$ & $25.41 \exp (2.18 \varepsilon / k T) /$ & No & No \\
& & $(1.41 \exp (2.18 \varepsilon / k T)-0.41)$ & & No \\
Guo et al. (GWL-I) [31] & $(4 \pi / 3)\left(\lambda^{3}-1\right) \rho \sigma^{3} \exp (\varepsilon / k T)$ & 14.07 & Yes & No \\
Guo et al. (GWL-II) [31] & $(4 \pi / 3)\left(\lambda^{3}-1\right) \rho \sigma^{3} \exp (\varepsilon / k T)$ & 25.61 & Yes & Yes \\
Yu-Chen (YC) [23] & $(4 \pi / 3)\left(\lambda^{3}-1\right) \rho \sigma^{3} \exp (\varepsilon / k T)$ & 20.4 & Yes & Yes \\
This work & $(4 \pi / 3)\left(\lambda^{3}-1\right) \rho \sigma^{3} \exp (\varepsilon / k T)$ & 18 & & \\
\hline
\end{tabular}


Table 2

Comparison of the calculated coordination numbers for pure SW fluids from various models

\begin{tabular}{|c|c|c|c|c|c|c|c|c|c|c|}
\hline \multirow[t]{2}{*}{$\varepsilon / k T$} & \multirow[t]{2}{*}{$\rho \sigma^{3}$} & \multirow[t]{2}{*}{ Data points } & \multicolumn{7}{|l|}{$\mathrm{AAD} \%{ }^{\mathrm{a}}$} & \multirow[t]{2}{*}{ Data sources } \\
\hline & & & Alder [6] & LS & GWL-I & GWL-II & $\mathrm{LC}$ & $\mathrm{YC}$ & This work & \\
\hline 0 & $0.1-0.8$ & 8 & 4.06 & 7.85 & 21.67 & 3.32 & 3.46 & 2.5 & 0.73 & [8] \\
\hline 0.25 & $0.1-0.8$ & 8 & 14.63 & 4.72 & 14.12 & 1.21 & 1.12 & 0.77 & 1.84 & [8] \\
\hline 0.5 & $0.1-0.8$ & 8 & 25.40 & 3.04 & 6.09 & 1.79 & 2.27 & 3.06 & 3.79 & [8] \\
\hline 0.75 & $0.1-0.8$ & 8 & 31.35 & 3.65 & 6.07 & 3.50 & 3.54 & 6.24 & 6.06 & [8] \\
\hline 1.0 & $0.1-0.8$ & 8 & 35.44 & 7.27 & 8.19 & 4.64 & 4.01 & 7.19 & 5.18 & [8] \\
\hline 0.5714 & $0.05-0.8$ & 6 & 28.59 & 2.77 & 5.46 & 2.45 & 2.23 & 2.76 & 3.85 & [31] \\
\hline 0.4 & $0.05-0.8$ & 6 & 21.83 & 4.65 & 8.15 & 1.95 & 2.38 & 2.11 & 3.26 & {$[31]$} \\
\hline 0.33 & $0.05-0.8$ & 6 & 18.51 & 5.80 & 9.52 & 1.74 & 2.18 & 1.77 & 2.99 & [31] \\
\hline Grand average & & 58 & 22.42 & 5.03 & 10.14 & 2.63 & 2.69 & 3.41 & 3.47 & \\
\hline
\end{tabular}

${ }^{\mathrm{a}} \mathrm{AAD} \%=\frac{100}{N} \sum\left[\frac{\left|N_{\mathrm{c}, \mathrm{MC}}-N_{\mathrm{c}}^{\mathrm{cal}}\right|}{N_{\mathrm{c}, \mathrm{MC}}}\right], N=$ total data point number.

According to the perturbation theory of Barker and Henderson [26], the radial distribution function of a SW fluid can be expressed in a logarithmic expansion form as

$$
g^{\mathrm{SW}}(\sigma)=g^{\mathrm{hs}}(\sigma) \exp \left[f\left(\lambda \sigma, \frac{\varepsilon}{k T}\right)\right]
$$

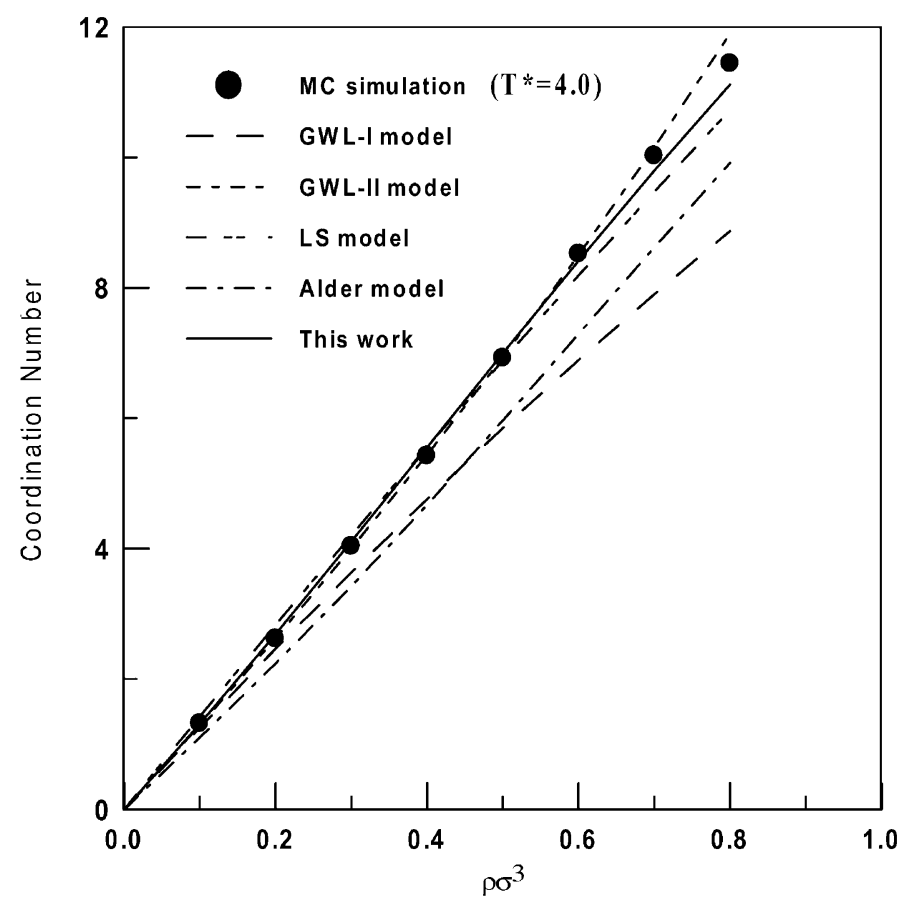

Fig. 1. Comparison of the calculated coordination numbers of pure SW fluids various models (MC computer simulation data: [8]). 
Table 3

Comparison of various models in the calculation of the compressibility factor of pure SW fluids

\begin{tabular}{|c|c|c|c|c|c|c|c|c|c|c|c|}
\hline \multirow{2}{*}{$\begin{array}{l}\text { Range } \\
\text { of } \varepsilon / k T\end{array}$} & \multirow{2}{*}{$\begin{array}{l}\text { Range of } \\
\rho \sigma^{3}\end{array}$} & \multirow{2}{*}{$\begin{array}{l}\text { Data } \\
\text { points }\end{array}$} & \multicolumn{8}{|c|}{$\mathrm{AAD} \%^{\mathrm{a}}$} & \multirow{2}{*}{$\begin{array}{l}\text { Data } \\
\text { sources }\end{array}$} \\
\hline & & & $\mathrm{LC}$ & LS & GWL-I & GWL-II & SL [33] & Alder & MPHS [28] & This work & \\
\hline $0.33-0.57$ & $0.05-0.80$ & 18 & 0.171 & 0.223 & 0.387 & 0.165 & 0.118 & 0.158 & 0.079 & 0.099 & [31] \\
\hline $0.09-1.92$ & $0.20-0.88$ & 145 & 0.674 & 0.119 & 0.303 & 0.368 & 0.367 & 0.218 & 0.269 & 0.253 & [6] \\
\hline $0.25-1.25$ & $0.50-0.80$ & 15 & 0.502 & 0.584 & 0.992 & 0.518 & 0.220 & 0.396 & 0.180 & 0.249 & [32] \\
\hline
\end{tabular}

${ }^{\mathrm{a}} \mathrm{AAD} \%=\frac{1}{N} \sum\left[\left|Z_{\mathrm{c}, \mathrm{MC}}-Z_{\mathrm{c}}^{\mathrm{cal}}\right|\right], N=$ total data point number.

where $g^{\mathrm{hs}}(\sigma)$ is the hard sphere distribution function at contact [21]

$$
g^{\mathrm{hs}}(\sigma)=\frac{2-\eta}{2(1-\eta)^{3}}
$$

Applying Eq. (17) through Eq. (19), the chain term contribution to the Helmholtz free energy is obtained. Differentiation of the Helmholtz free energy with respect to the reduced density yields the expression for the chain term of the compressibility factor

$$
z^{\text {chain }}=(1-m)\left[\frac{\eta(5-2 \eta)}{(1-\eta)(2-\eta)}+Z^{\text {corr }}(\lambda \sigma) \times F\left(\frac{\varepsilon}{k T}\right)\right]
$$

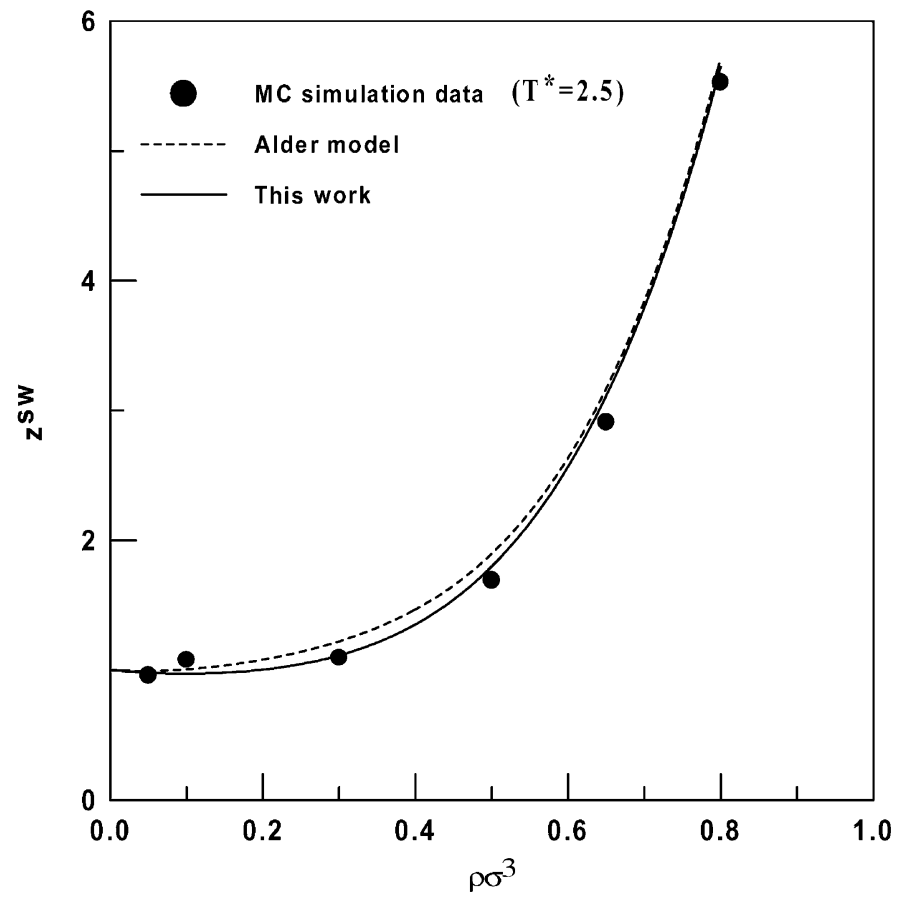

Fig. 2. Comparison of the calculated compressibility factors of pure SW fluids from various models (MC computer simulation data: [31]). 


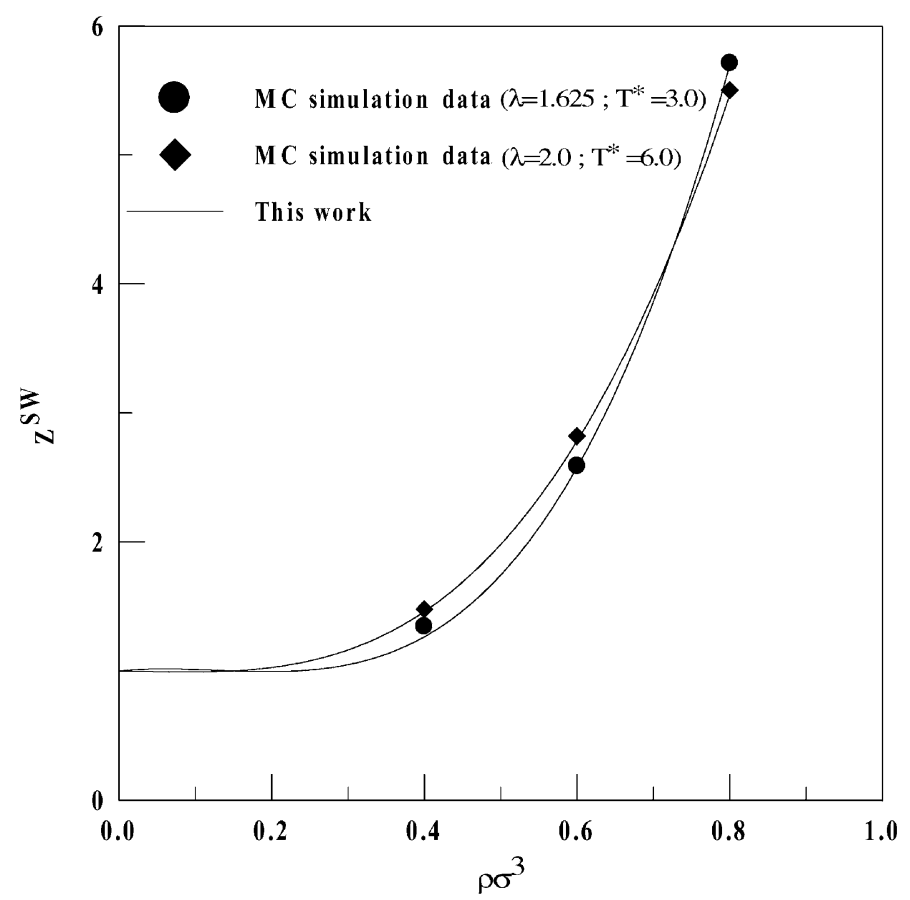

Fig. 3. Compressibility factors of the pure SW fluids calculated in the work at various $\lambda$ and $T^{*}$ values (MC computer simulation data: [31]).

The second term in the parenthesis on the right-hand side of Eq. (20) comes from the derivative of the perturbation terms of the $\mathrm{SW}$ radial distribution function with respect to the reduced density. Functional forms and parameters of $z^{\text {corr }}$ and $F$ are correlated using the molecular simulation data for the compressibility factors of SW chain fluids at various well widths and temperatures. The results are expressed as

$$
z^{\mathrm{corr}}=\frac{\left(1+5 \eta_{\mathrm{eff}}\right)\left(\eta_{\mathrm{eff}}+C_{2} \eta^{2}+C_{3} \eta^{3}\right)}{2\left(1+\eta_{\mathrm{eff}}\right)^{3}}
$$

where the effective reduced density is expressed by a polynomial parameterization

$$
\eta_{\mathrm{eff}}=C_{1} \eta+C_{2} \eta^{2}+C_{3} \eta^{3}
$$

The coefficients $C_{i}$ in Eq. (22) are regressed as functions of the well width for SW fluids

$$
\left(\begin{array}{l}
C_{1} \\
C_{2} \\
C_{3}
\end{array}\right)=\left(\begin{array}{ccc}
1.01217 & 1.41012 & -1.42229 \\
0.40337 & 3.47579 & -3.87916 \\
-4.27319 & 2.33210 & 1.94109
\end{array}\right)\left(\begin{array}{c}
1 \\
\lambda \\
\lambda^{2}
\end{array}\right)
$$

The temperature function $F$ is correlated by

$$
F\left(\frac{\varepsilon}{k T}\right)=\left\{18.64084\left(\frac{\varepsilon}{k T}\right)+21.67648\left(\frac{\varepsilon}{k T}\right)^{2}-4.19834\left(\frac{\varepsilon}{k T}\right)^{3}\right\}
$$


Table 4

Comparison of the calculated compressibility factors for square-well chains by different models

\begin{tabular}{lllllll}
\hline Segment number & Range of $T^{*}$ & Range of $\rho \sigma^{3}$ & Data points & \multicolumn{2}{c}{ AAD\% ${ }^{\text {a }}$} & \multirow{2}{*}{ Data sources } \\
\cline { 3 - 6 } & & & GFD & This work \\
\hline 4-mer & $1.5-4.0$ & $0.1-0.85$ & 34 & 0.162 & 0.175 & {$[24,34]$} \\
8 -mer & $1.5-4.0$ & $0.2-0.85$ & 29 & 0.091 & 0.075 & {$[24,35]$} \\
$16-$ mer & $1.5-4.0$ & $0.2-0.80$ & 22 & 0.107 & 0.106 & {$[24,35]$} \\
\hline
\end{tabular}

${ }^{\mathrm{a}} \mathrm{AAD} \%=\frac{1}{N} \sum\left[\frac{\left|Z_{\mathrm{c}, \mathrm{MC}}-Z_{\mathrm{c}}^{\mathrm{cal}}\right|}{Z_{\mathrm{c}, \mathrm{MC}}}\right], N=$ total data point number.

The complete chain term EOS, which is applicable for hard sphere to SW chains with variable well width and temperatures, is obtained by substituting Eqs. (21)-(24) into Eq. (20). Combining Eqs. (3), (14) and (20) into Eq. (2) gives the complete EOS for a pure SW chain fluid.

Table 4 shows the calculated results of the compressibility factors for the SW chains at various densities and temperatures. Our calculated results agree well with the molecular simulated data. The comparison of our results with those of the GFD EOS [36], which was derived on the basis of SW chain fluids as well, also yields good agreement. Fig. 4 shows the graphical comparison of our calculated results with the molecular simulation data of hard sphere chains. The agreement is satisfactory. Figs. 5-7 present our calculation results for SW chains at $\lambda=1.5$ and at various reduced temperatures. It is observed that our

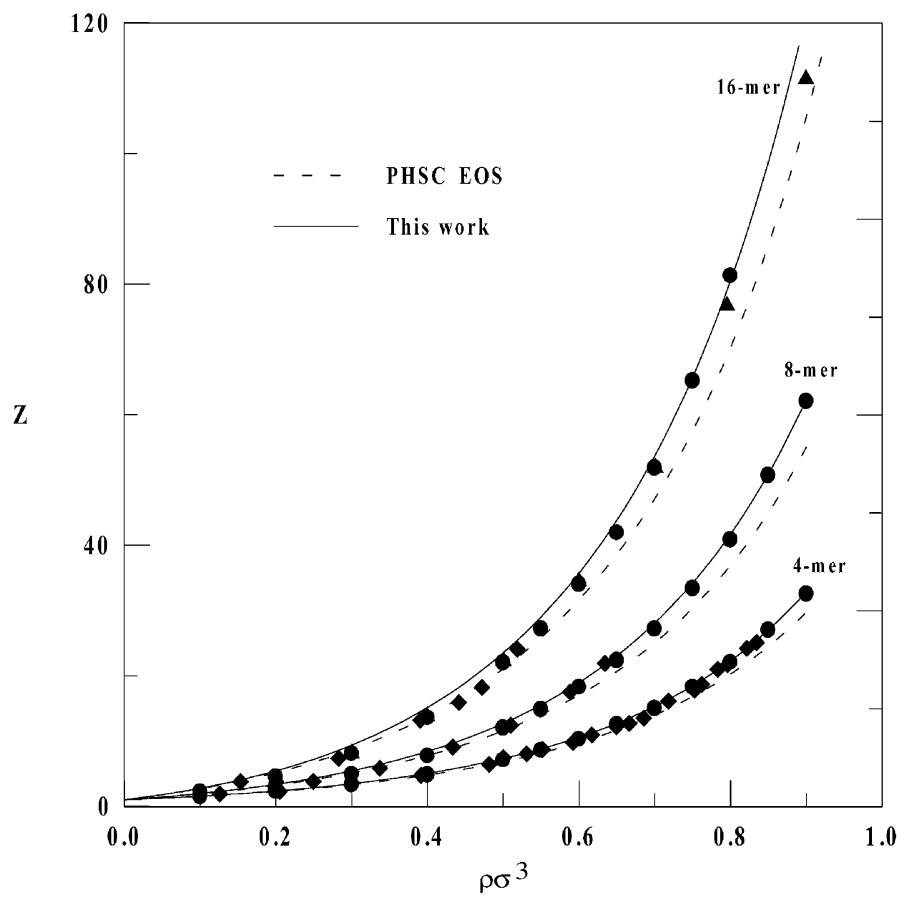

Fig. 4. Comparison of the compressibility factors of hard-sphere chains from this work and PHSC EOS [13] (MC computer simulation data: $(\boldsymbol{O})[36] ;(\diamond)$ [37]; (A) [38]). 


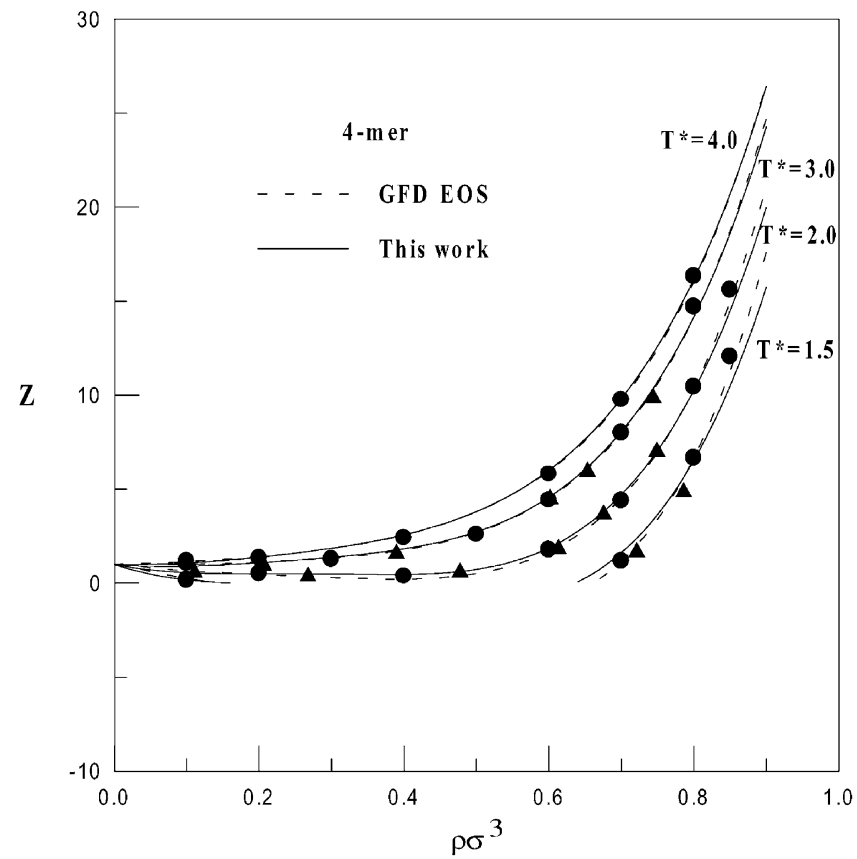

Fig. 5. Comparison of the compressibility factors of square-well 4-mer chains of $\lambda=1.5$ from this work and GFD EOS [35] (MC computer simulation data: $(\mathbf{O})[24] ;(\mathbf{\Delta})$ [34]).

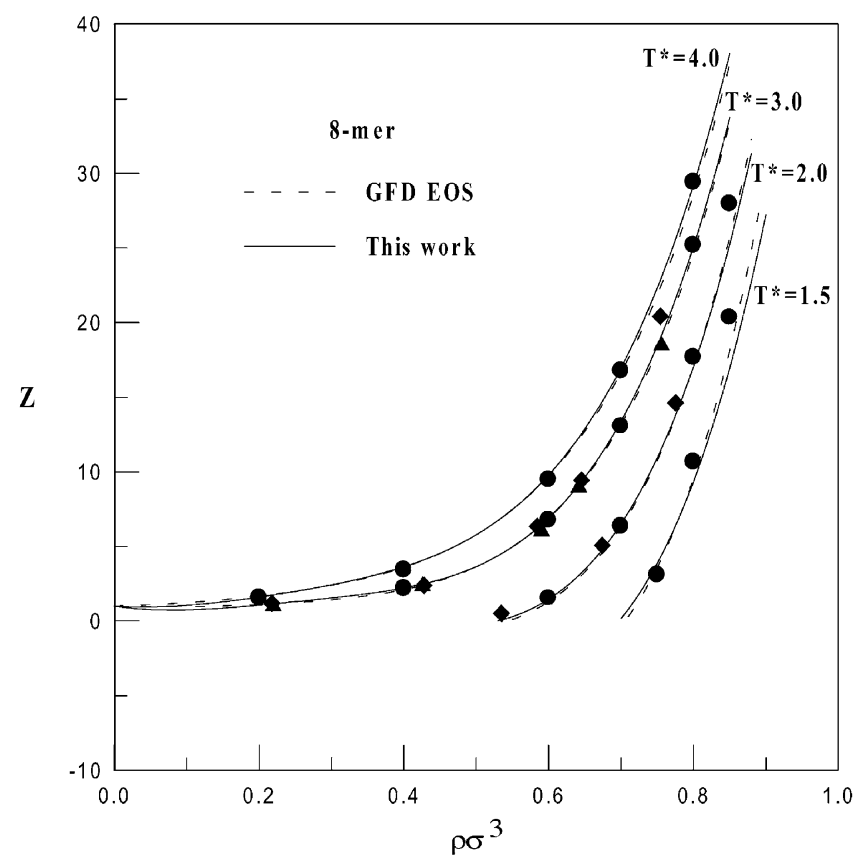

Fig. 6. Comparison of the compressibility factors of square-well 8-mer chains of $\lambda=1.5$ from this work and GFD EOS [35]

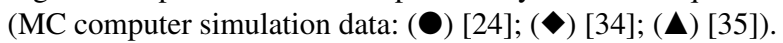




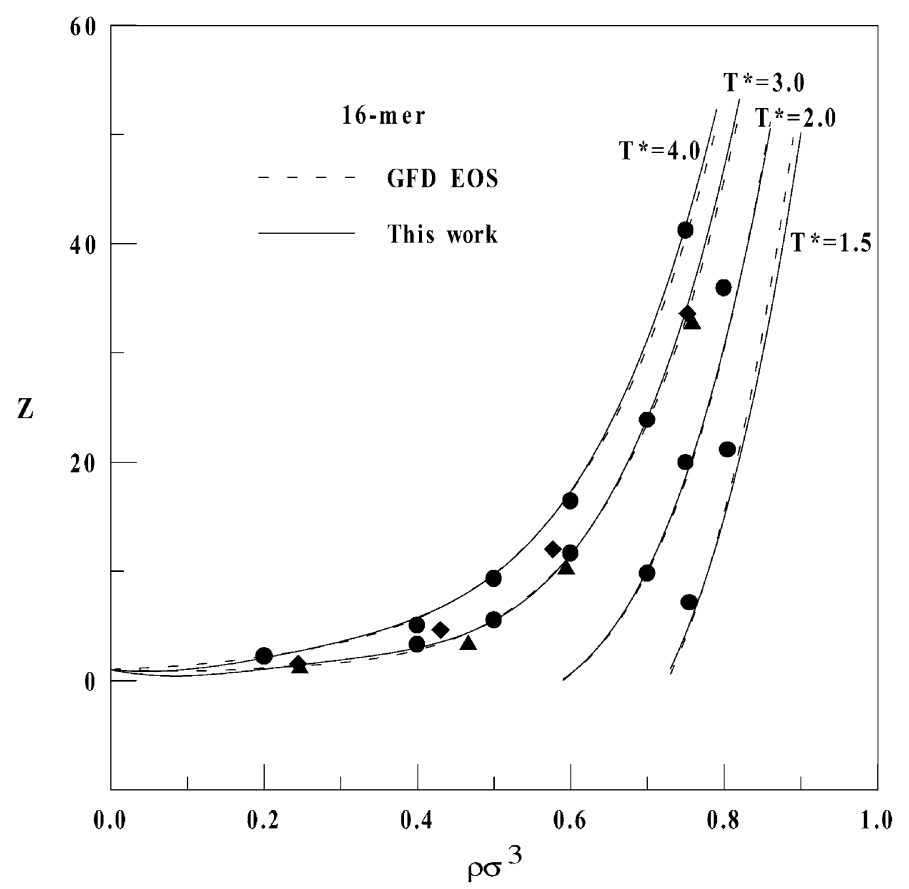

Fig. 7. Comparison of the compressibility factors of square-well 16-mer chains of $\lambda=1.5$ from this work and GFD EOS [35] (MC computer simulation data: $(\bullet)$ [24]; $(\bullet)$ [34]; (\) [35]).

results match well with the molecular simulation data for various SW chain fluids. The accuracy of this study is also comparable to that of the GFD EOS at all reduced conditions. Our EOS has been tested with the molecular simulation data for the SW monomer and chain fluids with satisfactory results. It is indicated that the complete EOS is theoretically consistent. It is also simple in mathematical formulation for engineering applications in phase equilibrium calculations of real fluid systems.

\section{Applications of the new EOS to real fluid systems}

The EOS developed in this study is applied in real fluid calculations. There are four parameters in the new EOS: the number of segments $m$, the width of the SW potential $\lambda$, the molar volume of a hard sphere $V^{0}$, and the interaction energy parameter $\varepsilon / k T$. These parameters for real pure fluids are obtained through correlation of the saturated properties. Two parameters of $\varepsilon / k T$ and $V^{0}$ are taken as temperature dependent

$$
\begin{aligned}
\frac{\varepsilon}{k T} & =\frac{\left(\varepsilon^{0} / k\right)-0.04}{T} \\
V^{0} & =V^{00}+0.005 T
\end{aligned}
$$

The vapor pressure and saturated liquid molar volume data of pure non-associating fluids are used to regress the four EOS parameters of $m, \lambda, \varepsilon^{0} / k T$ and $V^{00}$. Bubble point pressure calculations were carried 
out using the new EOS where the equal fugacity criterion is satisfied

$$
f_{i}^{\mathrm{V}}=f_{i}^{\mathrm{L}}
$$

The fugacity coefficient for a pure fluid is determined from this ES as

$$
\begin{aligned}
\ln f= & m\left(\frac{4 \eta-3 \eta^{2}}{(1-\eta)^{2}}\right)+m\left(\ln \frac{2-\eta}{2(1-\eta)^{3}}+\left(\frac{3}{2\left(1-\eta_{\mathrm{eff}}\right)^{2}}-\frac{5}{2\left(1-\eta_{\mathrm{eff}}\right)}+1\right) \times F\left(\frac{\varepsilon}{k T}\right)\right) \\
& \times m\left(\frac { 2 } { 3 } \pi ( \lambda ^ { 3 } - 1 ) \rho \sigma ^ { 3 } \left\{\left(\exp \left(\frac{\varepsilon}{k T}\right)-1.28\left(\frac{\varepsilon}{k T}\right)-1\right) \exp (1-\varphi)+1.28\left(\frac{\varepsilon}{k T}\right)\right.\right. \\
& \left.\left.+\Omega(1-\exp (1-\varphi))\left(\sqrt{2}-\rho \sigma^{3}\right)\right\}\right)-\ln Z+(Z-1)
\end{aligned}
$$

The optimized pure fluid EOS parameters are evaluated by minimizing the following objective function

$$
\mathrm{obj}=\frac{100}{N} \sum_{1}^{N}\left[\frac{\left|P_{i}^{\exp }-P_{i}^{\mathrm{cal}}\right|}{P_{i}^{\exp }}+\frac{\left|V_{i}^{\exp }-V_{i}^{\mathrm{cal}}\right|}{V_{i}^{\exp }}\right]
$$

Pure fluids of $n$-alkanes are calculated in this study, and the results are presented in Table 5. It is observed that the new EOS gives good correlation results for the saturated properties of $n$-alkanes up to $n$-eicosane. The AAD values of vapor pressure and saturated liquid molar volume are $<1 \%$. The pure fluid parameters also show satisfactory correlation with the molecular weights. These relationships are shown in Figs. 8-11. The correlation equations for these EOS parameters are

$$
\begin{aligned}
& m=0.01357 \mathrm{MW}+0.91007 \\
& m \lambda=0.02010 \mathrm{MW}+1.59172 \\
& m V^{00}=1.09404 \mathrm{MW}+9.44700 \\
& m\left(\frac{\varepsilon^{0}}{k}\right)=5.75551 \mathrm{MW}+37.82067
\end{aligned}
$$

Using these generalized EOS parameters, pure fluid properties are calculated and compared with the experimental data. Fig. 12 shows the calculated vapor pressures of some $n$-alkane molecules, and the agreement with experimental data is satisfactory. Fig. 13 presents that the calculated saturated liquid molar volumes match well with experimental data. The calculated saturated curves for ethane and $n$-octane are plotted in Fig. 14 as a function of temperature against the saturated density. Satisfactory results are observed up to the critical region. Although the EOS parameters are correlated from normal alkanes up to $n$-eicosane, we have further tested the generalized correlation equations of the EOS parameters by calculating the vapor pressures of $n$-alkanes with carbon numbers higher than 20. Fig. 15 shows our predicted vapor pressures for $n-\mathrm{C}_{22} \mathrm{H}_{46}, n-\mathrm{C}_{24} \mathrm{H}_{50}$ and $n-\mathrm{C}_{28} \mathrm{H}_{58}$ using the generalized EOS parameters. The predicted results agree quite well with the experimental data, and an AAD of $<1.0 \%$ in vapor pressure is obtained. The real fluid calculation results indicate that the new EOS is good for engineering applications. The generalized EOS parameters give satisfactory predictions of saturated properties for straight chain molecules. Extensions to fluid mixture calculations are currently undergoing. 
Table 5

Calculated results of saturated vapor pressures and liquid molar volumes of $n$-alkanes using the equation of state of this study

\begin{tabular}{|c|c|c|c|c|c|c|c|c|c|c|c|}
\hline \multirow[t]{2}{*}{ Compounds } & \multicolumn{2}{|c|}{ Temperature range (K) } & \multicolumn{2}{|c|}{ Data point } & \multirow[t]{2}{*}{$\lambda$} & \multirow[t]{2}{*}{$m$} & \multirow[t]{2}{*}{$V^{00}$} & \multirow[t]{2}{*}{$\varepsilon^{0} / k$} & \multicolumn{2}{|c|}{ This work } & \multirow{2}{*}{$\begin{array}{l}\text { Data } \\
\text { sources }\end{array}$} \\
\hline & $P^{\mathrm{V}}$ & $V^{\mathrm{L}}$ & $P^{\mathrm{V}}$ & $V^{\mathrm{L}}$ & & & & & $\begin{array}{l}\text { AAD } \\
P(\%)^{\mathrm{a}}\end{array}$ & $\begin{array}{l}\mathrm{AAD} \\
V^{\mathrm{L}}(\%)\end{array}$ & \\
\hline Methane & 100-190 & $100-185$ & 39 & 37 & 1.61178 & 1.12782 & 23.93887 & 115.40523 & 0.59 & 1.54 & [39] \\
\hline Ethane & $163-300$ & $163-285$ & 29 & 26 & 1.61008 & 1.36406 & 30.27320 & 168.45026 & 0.25 & 1.06 & [39] \\
\hline Propane & $173-357$ & $186-338$ & 27 & 24 & 1.60925 & 1.55972 & 36.70939 & 190.76833 & 1.09 & 0.99 & [39] \\
\hline$n$-Butane & $217-392$ & $217-392$ & 28 & 28 & 1.60726 & 1.65842 & 43.84412 & 215.80945 & 1.05 & 1.08 & [39] \\
\hline$n$-Pentane & $240-454$ & $240-446$ & 28 & 26 & 1.60084 & 1.80909 & 49.00159 & 234.85231 & 1.06 & 1.30 & [39] \\
\hline$n$-Hexane & $274-492$ & $274-485$ & 26 & 25 & 1.59123 & 1.95299 & 53.43706 & 254.67224 & 1.28 & 1.37 & [39] \\
\hline$n$-Heptane & $288-434$ & $288-483$ & 15 & 19 & 1.59013 & 2.23816 & 54.09795 & 255.05438 & 1.13 & 1.29 & [39] \\
\hline$n$-Octane & $320-480$ & $320-504$ & 19 & 24 & 1.58305 & 2.45614 & 55.14141 & 265.84300 & 1.46 & 0.92 & [39] \\
\hline$n$-Nonane & $312-503$ & $313-423$ & 32 & 12 & 1.57758 & 2.67085 & 56.05355 & 275.01950 & 1.05 & 0.28 & [40] \\
\hline$n$-Decane & $330-523$ & $333-443$ & 25 & 12 & 1.57054 & 2.90121 & 56.83758 & 284.95784 & 1.14 & 0.22 & [40] \\
\hline$n$-Undecane & $348-498$ & $353-473$ & 30 & 13 & 1.56440 & 3.10930 & 58.02339 & 295.01324 & 1.04 & 0.17 & [40] \\
\hline$n$-Dodecane & $364-520$ & $373-483$ & 30 & 12 & 1.55846 & 3.31484 & 59.01216 & 305.05256 & 1.10 & 0.14 & [40] \\
\hline$n$-Tridecane & $380-540$ & $393-513$ & 30 & 13 & 1.55350 & 3.50101 & 60.30446 & 314.63659 & 1.11 & 0.28 & [40] \\
\hline$n$-Tetradecane & $395-559$ & $413-523$ & 29 & 12 & 1.55129 & 3.65365 & 62.12625 & 321.74903 & 1.07 & 0.29 & [40] \\
\hline$n$-Pentadecane & $442-577$ & $423-543$ & 27 & 13 & 1.54767 & 3.82067 & 63.48757 & 329.89291 & 0.81 & 0.34 & [40] \\
\hline$n$-Hexadecane & $456-594$ & $443-553$ & 25 & 13 & 1.54206 & 4.00959 & 64.48757 & 339.93719 & 0.74 & 0.32 & [40] \\
\hline$n$-Heptadecane & $475-609$ & $453-573$ & 25 & 13 & 1.53652 & 4.19602 & 65.06363 & 350.02038 & 0.61 & 0.33 & [40] \\
\hline$n$-Octadecane & $493-624$ & $473-583$ & 24 & 12 & 1.53148 & 4.36444 & 66.01643 & 360.01967 & 0.63 & 0.33 & [40] \\
\hline$n$-Nonadecane & $505-638$ & $463-593$ & 25 & 14 & 1.52695 & 4.51191 & 67.11697 & 370.02789 & 0.76 & 0.30 & [40] \\
\hline$n$-Eicosane & $553-652$ & $473-613$ & 23 & 15 & 1.52234 & 4.65728 & 68.21162 & 380.33009 & 0.89 & 0.40 & [40] \\
\hline Grand average & & & 536 & 363 & & & & & 0.93 & 0.82 & \\
\hline
\end{tabular}

\footnotetext{
${ }^{\mathrm{a}} \mathrm{AAD} \%=\frac{100}{N} \sum\left[\frac{\left|M^{\exp }-M^{\mathrm{cal} \mid}\right|}{M^{\exp }}\right], M=P$ or $V^{\mathrm{L}}, N=$ total number of data points.
} 


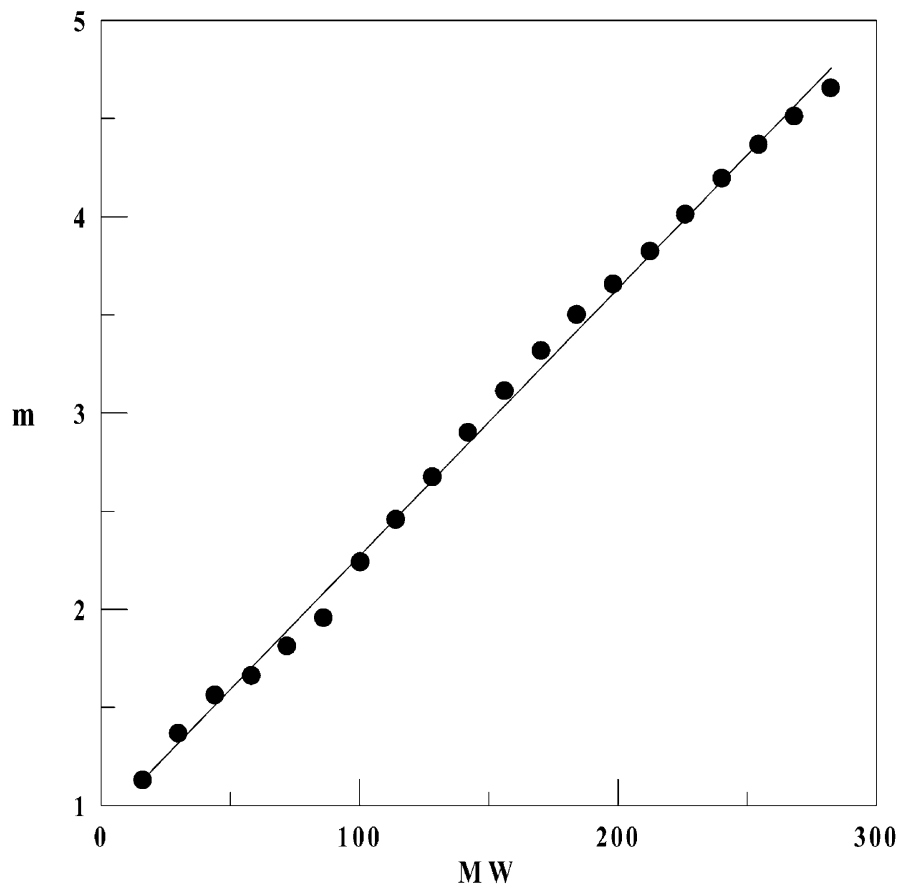

Fig. 8. Correlation of the segment number parameter $m$ in this work with the molecular weight of $n$-alkanes.

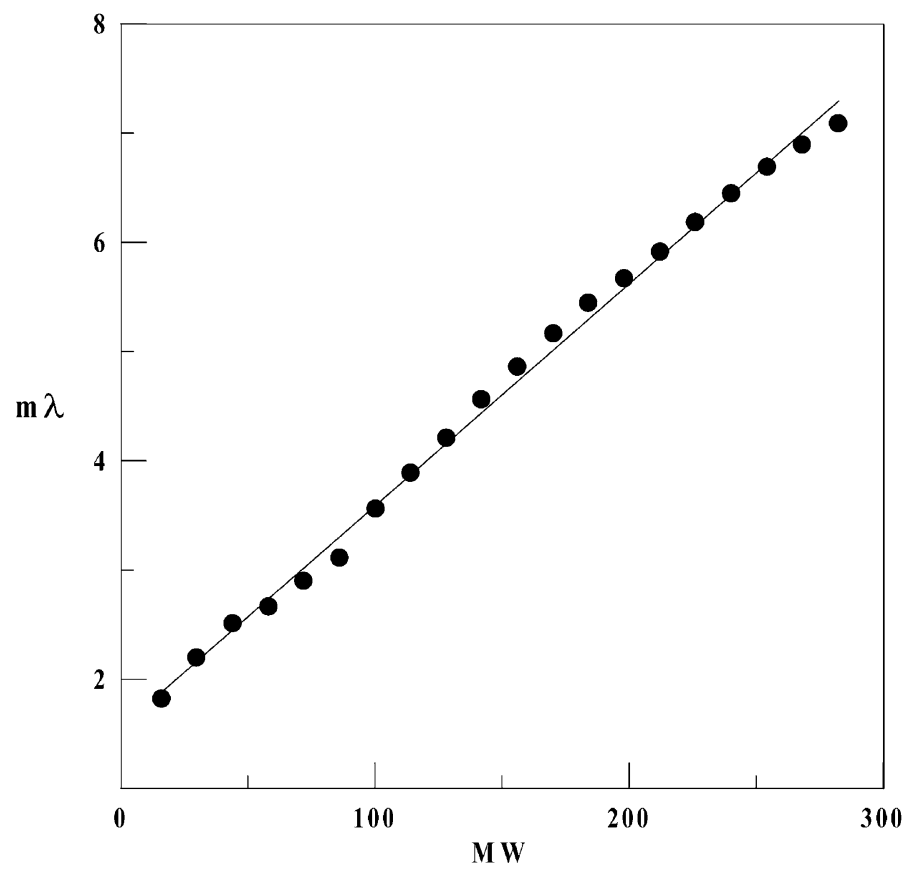

Fig. 9. Correlation of the $m \lambda$ parameters in this work with the molecular weight of $n$-alkanes. 


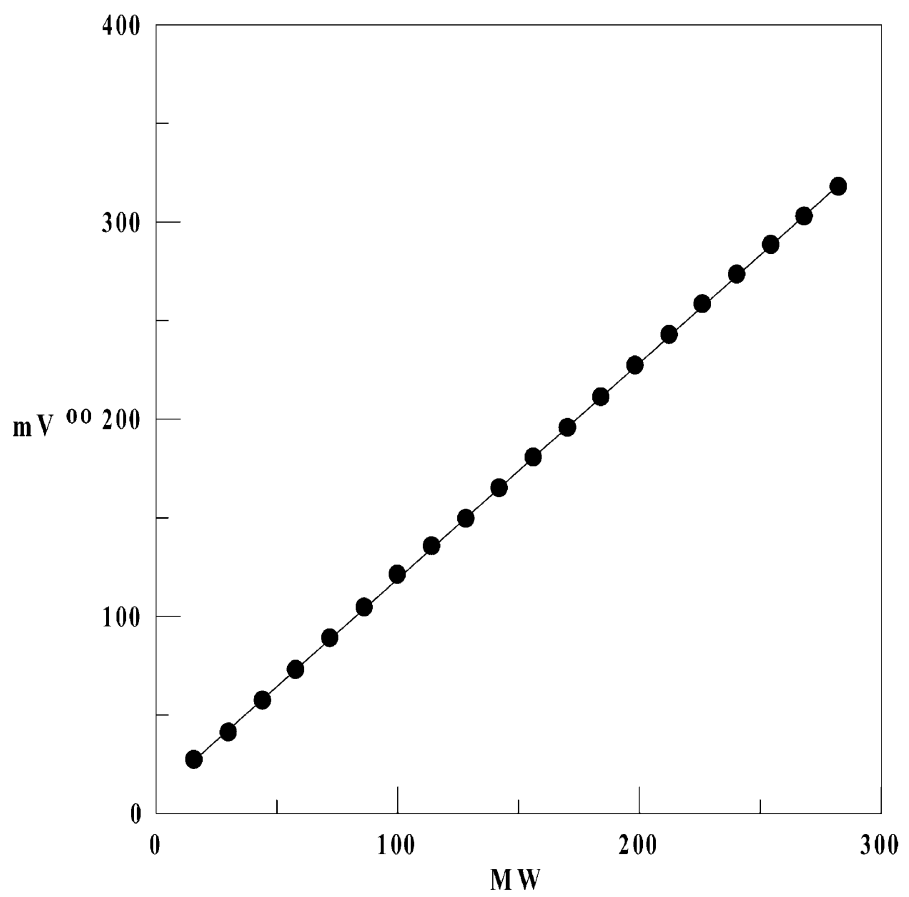

Fig. 10. Correlation of the $m V^{00}$ parameters in this work with the molecular weight of $n$-alkanes.

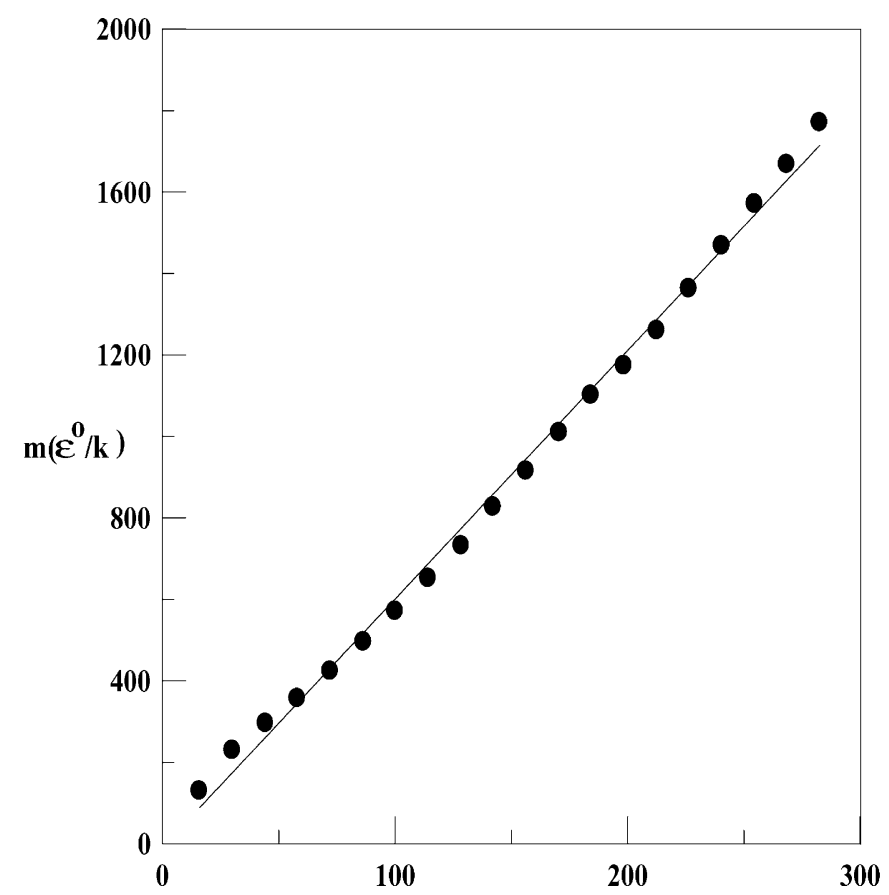

Fig. 11. Correlation of the $m\left(\varepsilon^{0} / k\right)$ parameters in this work with the molecular weight of $n$-alkanes. 


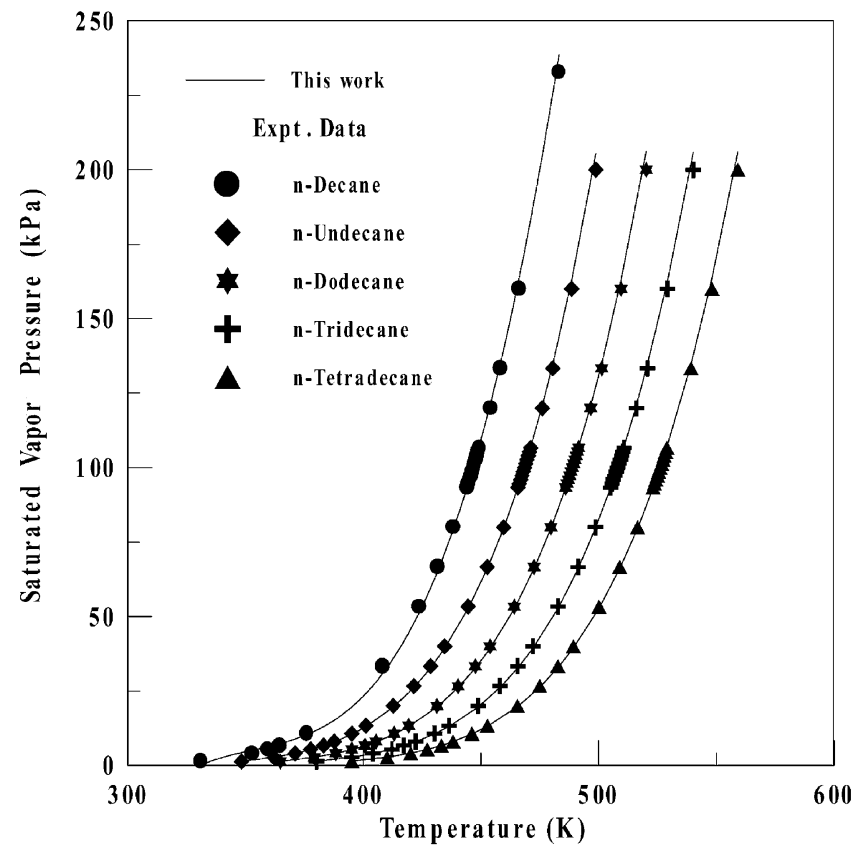

Fig. 12. Comparison of the calculated saturated vapor pressures of selected $n$-alkanes with the experimental data (data source: [40]).

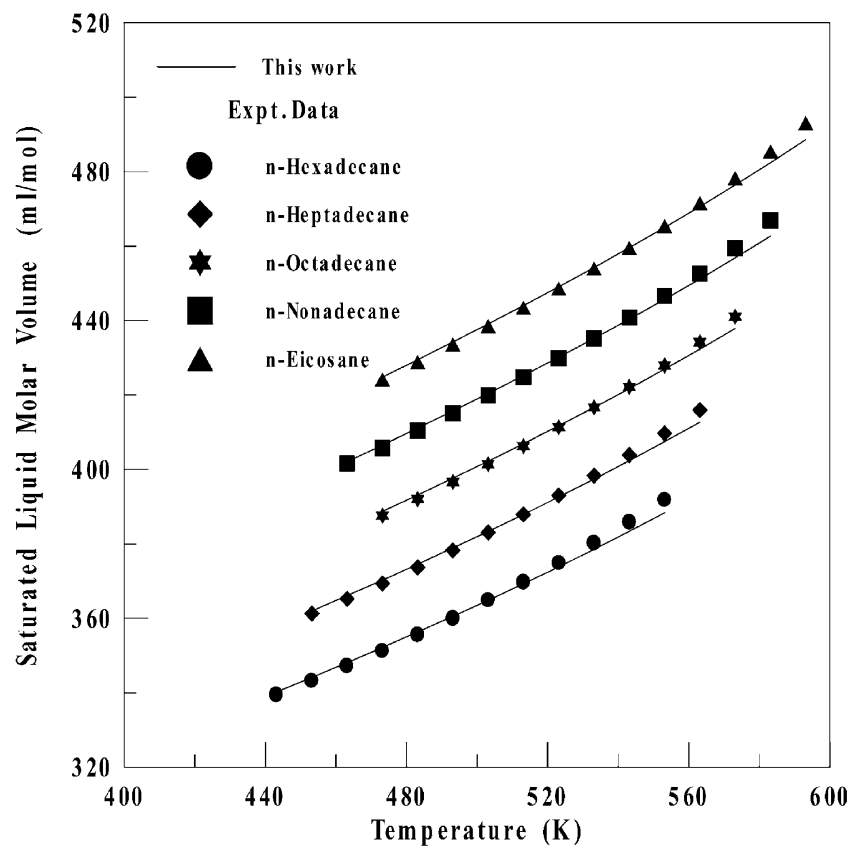

Fig. 13. Comparison of the calculated saturated liquid molar volumes of selected $n$-alkanes with the experimental data (data source: [40]). 


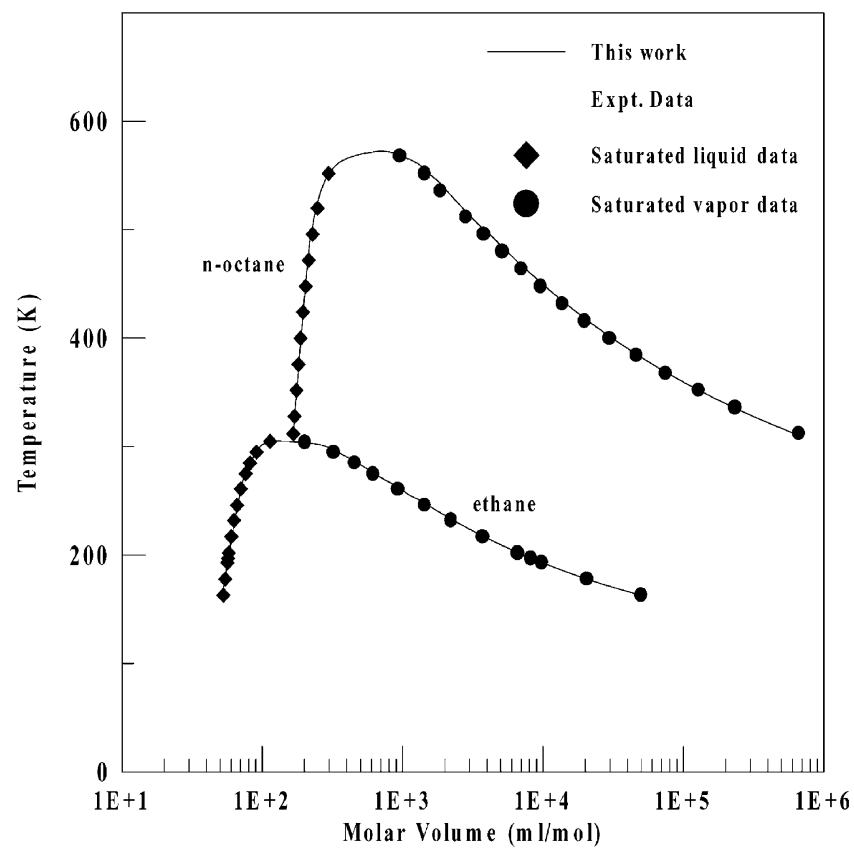

Fig. 14. Comparison of the calculated coexisting vapor and liquid densities for ethane and $n$-octane (data source: [39]).

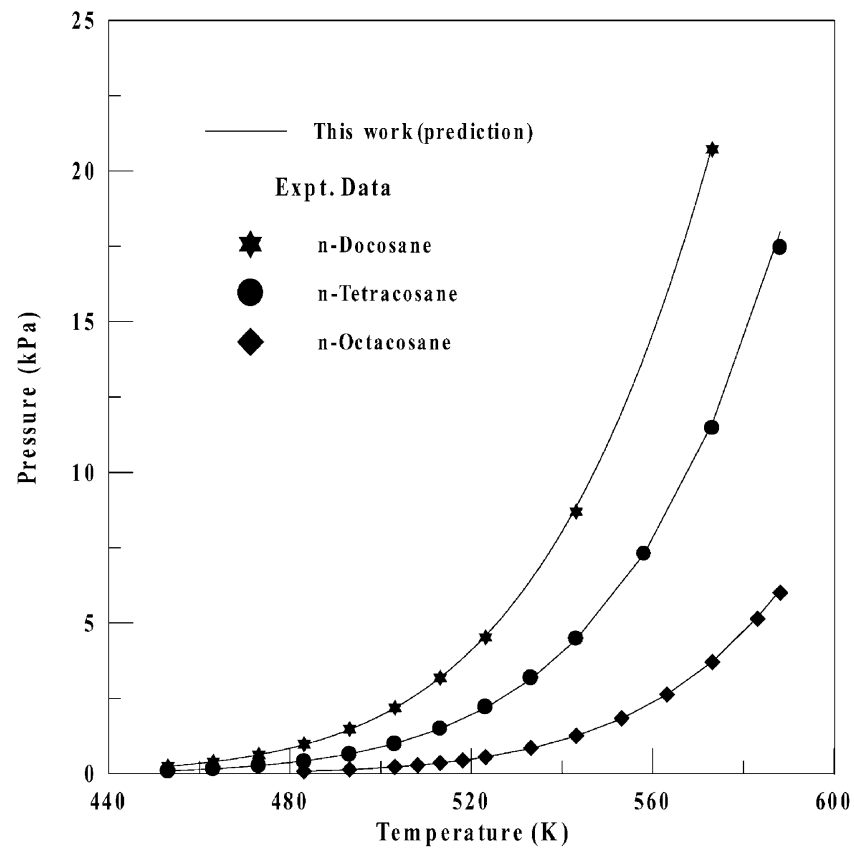

Fig. 15. Comparison of the predicted saturated vapor pressures of heavy $n$-alkanes with the experimental data (data source: [41]). 


\section{Conclusion}

A SAFT-based EOS is developed in this study for hard sphere and SW chain fluids. The dispersion term of the EOS is determined from a modified coordination number model that satisfies the theoretical requirements from low density to the close-packed limit. The chain contribution of the EOS is determined by correlating the perturbed terms with the molecular simulation data of the compressibility factors of SW chain fluids. A rather simpler mathematical form of the complete EOS is derived. The EOS is applicable to chain molecules and is tested for real $n$-alkanes in this study. Four EOS parameters show reasonable correlation with respect to the molecular weight of chain molecules. The generalized EOS shows good correlation results for the saturated properties for $n$-alkanes. The EOS also yields satisfactory predictions of the saturated properties for higher carbon number alkane molecules.

\section{List of symbols}

a Helmholtz free energy

A temperature function shown in Eq. (9)

$g \quad$ radial distribution function

$k \quad$ Boltzmann constant

$N \quad$ number of molecules

$N_{\mathrm{c}} \quad$ coordination number

$m \quad$ segment number

$P \quad$ pressure

$q \quad$ single particle partition function

$Q \quad$ canonical partition function

$R \quad$ distance parameter in the potential function

$T \quad$ temperature

$T^{*} \quad$ characteristic reduced temperature $(k T / \varepsilon)$

$V \quad$ volume of the system

$V_{\mathrm{f}} \quad$ free volume

$V^{0}, V^{00}$ molar volume parameters

$Z \quad$ compressibility factor

\section{Greek letters}

$\begin{array}{ll}\lambda & \text { well width of the square-well potential } \\ \Gamma & \text { potential function } \\ \varepsilon, \varepsilon^{0} & \text { energy parameter } \\ \pi & \text { mathematical constant } \\ \Phi & \text { mean potential } \\ \Psi & \text { correction term for the coordination number model } \\ \eta & \text { reduced density } \\ \Lambda & \text { de Broglie wavelength } \\ \rho & \text { number density } \\ \sigma & \text { segment diameter }\end{array}$




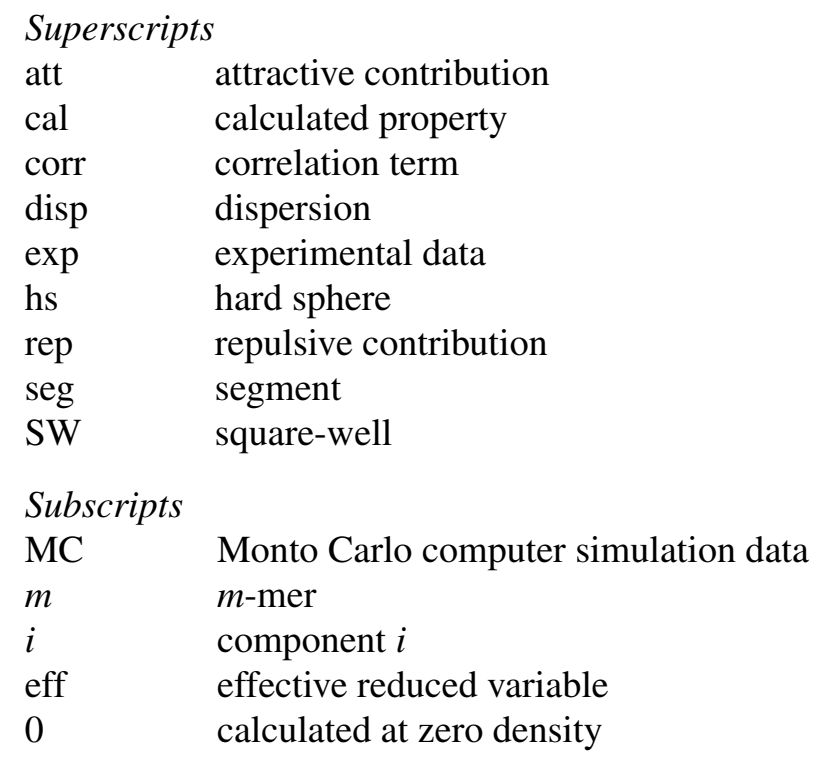

\section{Acknowledgements}

The authors are grateful to the National Science Council, Republic of China for supporting this research.

\section{References}

[1] M.S. Wertheim, J. Stat. Phys. 35 (1984a) 19-34.

[2] M.S. Wertheim, J. Stat. Phys. 35 (1984b) 35-47.

[3] M.S. Wertheim, J. Stat. Phys. 42 (1986a) 459-476.

[4] M.S. Wertheim, J. Stat. Phys. 42 (1986b) 477-492.

[5] S.H. Huang, M. Radosz, Ind. Eng. Chem. Res. 29 (1990) 2284-2294.

[6] B.J. Alder, D.A. Young, D.A. Mark, J. Chem. Phys. 56 (1972) 3013-3029.

[7] Y.H. Fu, S.I. Sandler, Ind. Eng. Chem. Res. 34 (1995) 1897-1909.

[8] K.H. Lee, M. Lombardo, S.I. Sandler, Fluid Phase Equilib. 21 (1985) 177-186.

[9] F.W. Tavares, J. Chang, S.I. Sandler, Fluid Phase Equilib. 140 (1997) 129-143.

[10] A. Gil-Villegas, A. Galindo, P.J. Whitehead, S.J. Mills, G. Jackson, J. Chem. Phys. 106 (1997) $4168-4186$.

[11] H. Adidharma, M. Radosz, Ind. Eng. Chem. Res. 37 (1998) 4453-4462.

[12] H. Adidharman, M. Radosz, Fluid Phase Equilib. 161 (1999) 1-20.

[13] Y. Song, S.M. Lambert, J.M. Prausnitz, Ind. Eng. Chem. Res. 33 (1994) 1047-1057.

[14] Y.C. Chiew, Mol. Phys. 70 (1990) 129-143.

[15] T. Hino, J.M. Prausnitz, Fluid Phase Equilib. 138 (1997) 105-130.

[16] J. Chang, S.I. Sandler, Mol. Phys. 81 (1994) 745-765.

[17] W. Feng, W. Wang, Ind. Eng. Chem. Res. 38 (1999) 4966-4974.

[18] I.H. Kim, Y.C. Bae, Fluid Phase Equilib. 168 (2000) 201-216.

[19] W.G. Chapman, G. Jackson, K.E. Gubbins, Mol. Phys. 65 (1988) 1057-1079.

[20] Y.S. Wei, R.J. Sadus, AIChE J. 46 (2000) 169-196.

[21] N.F. Carnahan, K.E. Starling, J. Chem. Phys. 51 (1969) 635-636. 
[22] S.S. Chen, A. Kreglewski, Ber-Bunsen-Ges Phys. Chem. 81 (1977) 1048-1052.

[23] M.L. Yu, Y.P. Chen, Fluid Phase Equilib. 111 (1995) 37-51.

[24] F.W. Tavares, J. Chang, S.I. Sandler, Mol. Phys. 86 (1995) 1451-1471.

[25] H.C. Andersen, D. Chandler, J. Chem. Phys. 57 (1972) 1918-1929.

[26] J.A. Barker, D. Henderson, Rev. Modern Phys. 48 (1976) 587-671.

[27] K.H. Lee, S.I. Sandler, N.C. Patel, Fluid Phase Equilib. 25 (1986) 31-49.

[28] M.L. Yu, Y.P. Chen, Fluid Phase Equilib. 129 (1997) 21-35.

[29] S.I. Sandler, Fluid Phase Equilib. 19 (1985) 233-257.

[30] R.J. Lee, K.C. Chao, Mol. Phys. 61 (1987) 1431-1442.

[31] M.X. Guo, W.C. Wang, H.Z. Lu, Fluid Phase Equilib. 60 (1990a) 37-45.

[32] D. Henderson, W.G. Madden, D.D. Fitts, J. Chem. Phys. 64 (1976) 5026-5034.

[33] S. Shen, B.C.-Y. Lu, Fluid Phase Equilib. 84 (1993) 9-22.

[34] A. Yethiraj, C.K. Hall, J. Chem. Phys. 95 (1991) 1999-2005.

[35] A. Yethiraj, C.K. Hall, J. Chem. Phys. 95 (1991) 8494-8506.

[36] J. Chang, S.I. Sandler, Chem. Eng. Sci. 49 (1994) 2777-2791.

[37] R. Dickman, C.K. Hall, J. Chem. Phys. 89 (1988) 3168-3174.

[38] J. Gao, J.H. Weiner, J. Chem. Phys. 91 (1989) 3168-3173.

[39] B.D. Smith, R. Srivastava, Thermodynamic Data for Pure Compounds, Elsevier, Amsterdam, 1986.

[40] F.D. Rossini, K.S. Pitzer, R.L. Arnett, R.M. Braun, G.C. Pimental, Selected Values of Properties of Hydrocarbons and Related Compound, API Project 44, Carnegie, Pittsburgh, 1952.

[41] D.L. Morgan, Ph.D. Thesis, Rice University, Houston, TX, April 1990. 